\title{
Procura por serviços ou profissionais de saúde entre adolescentes: um estudo multinível
}

\author{
Demand for health services or professionals among adolescents: \\ a multilevel study
}

\author{
Alisse Maria Chaves de Lima Peixoto (https://orcid.org/0000-0003-2430-6031) ${ }^{1}$ \\ Thaise Queiroz de Melo (https://orcid.org/0000-0002-1314-0155) ${ }^{2}$ \\ Livia Almeida Amaral Ferraz (https://orcid.org/0000-0002-9665-8450) ${ }^{3}$ \\ Carolina da Franca Bandeira Ferreira Santos (https://orcid.org/0000-0002-7365-2806) ${ }^{2}$ \\ Fabiana de Godoy (https://orcid.org/0000-0002-1946-9605) ${ }^{2}$ \\ Paula Andréa de Melo Valença (https://orcid.org/0000-0002-1433-5632) ${ }^{2}$ \\ Valdenice Aparecida de Menezes (https://orcid.org/0000-0003-4183-3239) ${ }^{4}$ \\ Viviane Colares (https://orcid.org/0000-0003-2912-2100) ${ }^{2}$
}

${ }^{1}$ Secretaria Municipal de Saúde do Recife. Av. Cais do Apolo 925, Centro. 50030-903 Recife PE Brasil. alissepeixoto@gmail.com

${ }^{2}$ Programa de Pós-

Graduação em Hebiatria, Universidade de Pernambuco. Recife PE Brasil.

${ }^{3}$ Programa de PósGraduação em Biologia Celular e Molecular Aplicada, Universidade de Pernambuco. Recife PE Brasil.

${ }^{4}$ Programa de Pós-

Graduação em Odontologia, Universidade de Pernambuco. Recife PE Brasil.

\begin{abstract}
This study aimed to analyze the demand for health services or professionals by adolescents with individual determinants and contextual variables (school teaching modality and Social Vulnerability Index). Data were collected from March to June 2018, in state public schools in Olinda, Pernambuco, Brazil, through a structured questionnaire with questions from the Youth Risk Behavior Survey (YRBS) and the National School Health Survey (PeNSE). Among the 2,454 adolescents, the proportion of those looking for a health service was higher among women; in adolescents whose mothers had a minimum of nine schooling years; in the physically active; and whose parents did not receive a family aid (Bolsa Familia). We observed that the contextual variables did not influence the demand for health services among adolescents. In the third model of the multilevel analysis, which analyzed the individual variables, we observed that only females $(O R=1,80)$, having a mother with schooling greater than or equal to 9 years of study $(O R=1,30)$, not receiving Bolsa Família $(O R=1,23)$, and physically active $(O R=1,32)$ were associated with an increased demand for health services or professionals.

Key words Adolescent, Health services accessibility, Adolescent health services, Patient health care acceptance
\end{abstract}

Resumo O objetivo deste estudo foi analisar a procura por serviços ou profissionais de saúde pelos adolescentes com determinantes individuais $e$ com variáveis contextuais, modalidade de ensino da escola e Índice de Vulnerabilidade Social. Os dados foram coletados no período de março a junho de 2018, em escolas públicas estaduais de Olinda, Pernambuco, através de um questionário estruturado com questões do Youth Risk Behavior Survey (YRBS) e da Pesquisa Nacional de Saúde do Escolar (PeNSE). Dentre os 2.454 adolescentes, a proporção daqueles que procuram por um serviço de saúde foi maior: no sexo feminino; em adolescentes cujas as mães tivessem escolaridade minima de nove anos; naqueles fisicamente ativos; e cujos responsáveis não recebiam Bolsa Família. Observou-se que as variáveis contextuais não influenciaram a procura por serviços de saúde entre os adolescentes. No terceiro modelo da análise multinivel, que analisou as variáveis individuais, observou-se que apenas o sexo feminino $(\mathrm{OR}=1,80)$, ter mãe com escolaridade maior ou igual a nove anos de estudo $(O R=1,30)$, não receber Bolsa Família $(O R=1,23)$ e ser ativo fisicamente $(O R=1,32)$ foram associados a uma maior procura por serviços ou profissionais de saúde.

Palavras-chave Adolescente, Acesso aos serviços de saúde, Serviços de saúde do Adolescente, Aceitação pelo paciente de cuidados de saúde 


\section{Introdução}

No Brasil, existem aproximadamente 45 milhões de adolescentes, muitos apresentam baixos indicadores sociais, principalmente na Região Nordeste $^{1}$. Foi observado na Pesquisa Nacional de Saúde que essa é uma das faixas etárias que menos procura por atendimento de saúde, seja para prevenção ou para recuperação da sua saúde ${ }^{2}$. Soma-se a isso, o fato dos adolescentes entre 15 a 19 anos apresentarem várias condutas de risco, como envolvimento em causas externas, uso abusivo de drogas e comportamento sexual de risco ${ }^{1}$.

O uso dos serviços de saúde é resultante de uma interação entre o comportamento do indivíduo em procurar por um serviço e do profissional que o conduz dentro do serviço. É, então, conceituado como quando há o contato direto com os serviços através de consultas, hospitalizações, assim como quando há o contato indireto, por realização de exames, por exemplo ${ }^{3}$. Estudos no Brasil e no mundo têm pesquisado sobre a procura e a utilização dos serviços de saúde, entretanto, a maioria analisa a associação apenas com variáveis individuais, como sexo, renda, escolaridade, raça/cor (referência de vários estudos). Entretanto, torna-se importante também o estudo da associação com variáveis contextuais, através de análise multinível.

Esse modelo multinível considera que indivíduos pertencentes a um mesmo grupo, como bairros, escolas ou famílias, são influenciados por estímulos semelhantes. Dessa forma, para se analisar o comportamento humano, é importante considerar tanto as características individuais como as características do contexto em que estão inseridos. Assim, consideram-se variáveis preditoras de diferentes níveis. Para esta análise, é imprescindível que as variáveis de cada nível sejam selecionadas, e tenham como base um modelo teórico ou empírico que justifique a sua participação $0^{4}$.

Diversos modelos teóricos explicativos têm estudado a relação entre a utilização dos serviços de saúde e seus determinantes. Dentre eles, há o modelo comportamental de Andersen, inicialmente desenvolvido em 1968, que considera como fatores determinantes do uso dos serviços de saúde: fatores de predisposição (ligados ao fato do indivíduo estar susceptível ao uso dos serviços de saúde, que podem ser os fatores demográficos, a estrutura social e as crenças em saúde); fatores capacitantes (relacionam-se à possibilidade de acesso, englobando tanto fatores individuais e familiares, quanto a oferta de serviços); e as necessidades de saúde, que incluem o estado de saúde dos indivíduos, autopercebido ou identificado por um profissional ${ }^{5}$.

Apesar da diferença existente entre os conceitos de uso e procura por serviços de saúde, os determinantes do uso dos serviços, descritos pelo modelo comportamental de Andersen, também conseguem explicar a procura pelos serviços de saúde. Isto porque o conceito de acesso, para este autor, está ligado à entrada inicial do indivíduo no sistema de saúde ${ }^{6}$.

Assim, a identificação dos fatores que influenciam a procura pelos serviços de saúde pelos adolescentes é fundamental para que a prática dos profissionais de saúde e a organização da rotina dos serviços estejam em conformidade com esse público. Desse modo, o objetivo deste estudo foi analisar a associação da procura por serviços ou profissionais de saúde pelos adolescentes com determinantes individuais (fatores predisponentes, fatores capacitantes e necessidades em saúde) e com variáveis contextuais (regime escolar e Índice de Vulnerabilidade Social).

\section{Métodos}

\section{Desenho do estudo e amostragem}

Trata-se de um estudo transversal, de caráter analítico com base escolar, que faz parte do projeto integrado: "Saúde bucal e comportamentos de risco à saúde modificáveis na adolescência acompanhar para prevenir".

O estudo foi realizado em Olinda, Pernambuco, com estudantes adolescentes de ambos os sexos, com idades entre 14 e 19 anos, matriculados em escolas da rede pública estadual do município.

A amostra foi calculada por meio do software estatístico Epidemiologic Open Source Statistics for Public Health (OpenEpi 3.02). O cálculo considerou o projeto maior. Assim, utilizou-se os dados do estudo piloto para as associações entre os parâmetros de pobre saúde bucal e os comportamentos de risco ou agravos para a saúde do adolescente. A maior estimativa amostral calculada foi adotada. Considerando-se um poder de 80\%, um nível de confiança de $95 \%$ e um Odds Ratio de 1.4 a amostra foi estimada em 1532. Foi adicionado um efeito de desenho de 1.2 para corrigir amostragem em cluster, assim como, foi adicionado $20 \%$ para minimizar possíveis perdas. A amostra final estimada foi de 2.206 adolescentes.

Em Olinda havia 39 escolas em 2018, dessas, oito possuíam turmas apenas de Ensino Funda- 
mental ou no turno da noite, assim, 31 escolas que contavam com 269 turmas e 9.126 estudantes eram elegíveis para a pesquisa.

Uma seleção amostral por conglomerado em dois estágios foi planejada, através do sorteio das escolas e das turmas. Entretanto, à medida que os estudantes das escolas e turmas sorteadas apresentavam o Termo de Consentimento Livre e Esclarecido (TCLE), e se verificava que não era suficiente, eram sorteadas outras escolas dentro da mesma Região Político Administrativa (RPA), sempre respeitando a proporcionalidade. Dessa forma, todas as 31 escolas foram visitadas, das quais, 27 concordaram com a realização da pesquisa.

Foram distribuídos TCLE para 7.255 estudantes que estavam presentes nos dias da distribuição. Desses, 37\% (2.700), distribuídos em 188 turmas, apresentaram os TCLE assinados por eles e pelos pais/responsáveis (Figura 1).

\section{Coleta de dados}

Os dados foram coletados no período de março a junho do ano de 2018. O questionário foi aplicado em uma sala de aula disponibilizada pela escola, sem a presença dos professores, com

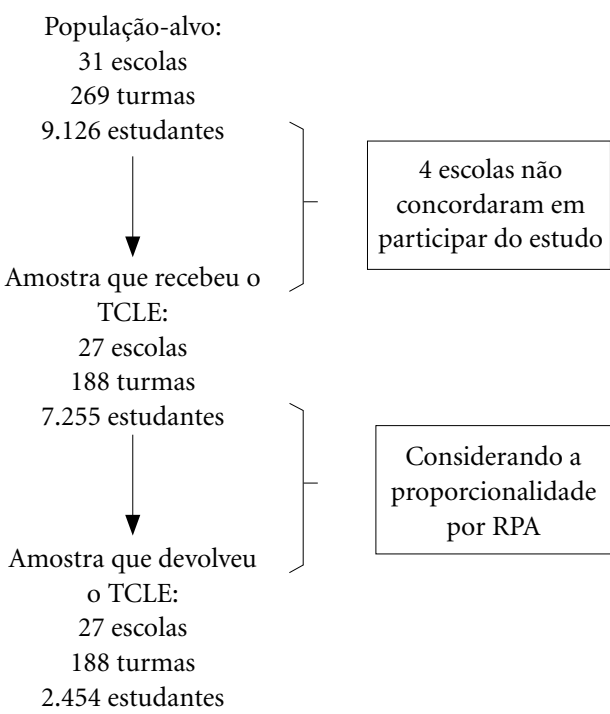

Figura 1. Fluxograma do processo de amostragem.

Fonte: elaborada pelas autoras. todos os alunos presentes no dia da coleta, que possuíam o TCLE assinado. A aplicação do questionário foi realizada por um pesquisador previamente treinado e teve uma duração aproximada de 30 minutos.

$\mathrm{O}$ instrumento utilizado na pesquisa foi $\mathrm{o}$ Youth Risk Behavior Survey (YRBS) validado no Brasil por Guedes e Lopes em 2007, proposto pelo Centro de Controle e Prevenção de Doenças (CDC) dos Estados Unidos, que faz parte de um Sistema de vigilância que monitora os comportamentos relacionados à saúde. Além do YRBS, algumas questões relacionadas à saúde presentes no questionário da Pesquisa Nacional de Saúde do Escolar (PeNSE) $)^{8}$ também foram utilizadas. Ao todo, o questionário da presente pesquisa foi composto por 119 questões. Para o presente estudo foram utilizadas seis questões referentes ao módulo socioeconômico e demográfico: faixa etária (14-16 / 17-19); sexo (masculino/feminino); escolaridade da mãe (menor que 9 anos de estudo/ maior ou igual a 9 anos de estudo); raça/ cor (branca/não branca); bolsa família (sim/ não). Uma questão sobre procura por serviços ou profissionais de saúde nos últimos 12 meses (sim/não) e uma questão sobre autopercepção de saúde (nada saudável ou não muito saudável/ saudável, bastante saudável ou completamente saudável), ambas presente na PeNSE. As condutas de risco à saúde foram avaliadas através das seguintes questões do YRBS: uso de álcool em binge - ato de beber grande quantidade de álcool de uma só vez - nos últimos 30 dias (sim/não); uso de cigarro nos últimos 30 dias (sim/não); número de parceiros sexuais (um ou nenhum/ dois ou mais); prática de atividade física - trezentos minutos por semana - (sim/não).

\section{Variáveis estudadas}

A variável dependente foi a procura por serviços ou profissionais de saúde nos 12 meses anteriores à pesquisa. As variáveis independentes incluídas no primeiro nível foram selecionadas com base no Modelo Comportamental de Andersen ${ }^{5}$. Os fatores predisponentes utilizados foram: demográficos (idade, sexo), estrutura social (escolaridade materna e raça) e crenças em saúde (consumo de álcool, tabaco, número de parceiros sexuais e prática de atividade física). $\mathrm{O}$ fator capacitante foi a adesão ao Bolsa Família, programa de transferência direta de renda, direcionado às famílias em situação de pobreza e de extrema pobreza em todo o País. A população-alvo do programa é constituída por famílias em situação 
de pobreza ou extrema pobreza. As famílias extremamente pobres são aquelas que têm renda mensal de até $\mathrm{R} \$ 89,00$ por pessoa. As famílias pobres são aquelas que têm renda mensal entre R \$ 89,01 e R \$ 178,00 por pessoa. As famílias pobres participam do programa, desde que tenham em sua composição gestantes e crianças ou adolescentes entre 0 e 17 anos.

A necessidade em saúde foi avaliada por meio da autopercepção de saúde. O segundo nível foi composto pelas 27 escolas que os estudantes frequentavam. Assim, uma das variáveis presentes no segundo nível correspondiam ao regime de ensino, que podia ser regular (20 horas semanais), semi-integral (32 horas semanais) ou integral (40 horas semanais) ${ }^{9}$.

O processo de ampliação do regime de ensino escolar teve início em Pernambuco em 2008 e faz parte do Programa de Educação Integral, política pública voltada para a melhoria do Ensino Médio. Esta política considera que o regime integral vai além da ampliação do tempo na escola, pois implica no desenvolvimento das diversas dimensões do ser humano'.

Além do regime escolar, outra variável inserida no segundo nível do modelo multinível foi o Índice de Vulnerabilidade Social (IVS), que se baseia no conceito de vulnerabilidade social e parte do reconhecimento de que essas vulnerabilidades decorrem de processos sociais amplos, dos quais os indivíduos sozinhos não têm meios para mudar. Apenas o Estado, através de políticas públicas, tem condições de alterar ${ }^{10}$.

Ele adota 16 indicadores presentes no bloco de vulnerabilidade social do Atlas do Desenvolvimento Humano $(\mathrm{ADH})$, subdivididos em três dimensões: infraestrutura urbana do território; capital humano dos domicílios deste território; renda, acesso aos trabalho e forma de inserção, formal ou não. Quanto mais alta a vulnerabilidade social em determinado território, o IVS tem valores mais próximos a um. Valores mais próximos a zero indicam baixa ou inexistente vulnerabilidade ${ }^{10}$.

Assim, o IVS da Unidade de Desenvolvimento Humano (UDH) na qual cada escola fazia parte foram localizados através do mapa do Instituto de Pesquisa Econômica Aplicada (IPEA) ${ }^{10}$.

\section{Análise estatística}

Os dados foram digitados com dupla entrada no EpiData 3.1. As análises foram realizadas no software Stata 15.1. Frequências absoluta e relativa foram empregadas para descrever os par- ticipantes do estudo. Teste de qui-quadrado foi empregado para avaliar associações bivariadas entre as variáveis independentes e a procura por serviços de saúde. Para testar a associação das variáveis individuais e contextuais com a procura por serviços de saúde, foi realizada uma análise multinível no modelo logístico misto (efeito fixo com intercepto randômico).

Foram ajustados modelos multinível logísticos, com indivíduos como primeiro nível e as escolas como segundo. Os modelos foram ajustados primeiramente sem a inclusão de variáveis independentes (modelo vazio), para testar a variância inicial atribuível às escolas. Na sequência, foram ajustados modelos com as variáveis contextuais e depois com as individuais que apresentaram um valor de $p$ menor ou igual a 0,05 na análise bivariada. No quarto e último modelo, foi testada a associação entre as variáveis individuais e contextuais ao mesmo tempo.

A heterogeneidade entre as escolas foi calculada, em todos os modelos, através do Median Odds Ratio (MOR) ${ }^{11}$. Além disso também foram calculadas as mudanças proporcionais na variância $(\mathrm{PCV})^{12}$.

O projeto maior foi submetido e aprovado no Comitê de Ética em Pesquisa da Universidade de Pernambuco, conforme preconizado pela Comissão Nacional de Ética em Pesquisa (CONEP) através da Resolução do Conselho Nacional de Saúde nº 466/2012.

\section{Resultados}

No total, a amostra foi formada por 2.454 adolescentes, 47 foram excluídos, 46 porque não responderam a pergunta correspondente à variável dependente desta pesquisa e um porque estava fora da faixa etária de 14 a 19 anos. A maioria dos participantes do sexo feminino $(55,87 \%)$ e na faixa etária de 14 a 16 anos (52,70\%), e a maioria das mães tinha menos de 9 anos de escolaridade. Ao serem questionados sobre sua cor de pele, $16,70 \%$ dos adolescentes relataram serem brancos. Poucos adolescentes relataram o uso de cigarros (10,83\%). Por outro lado, $32,04 \%$ assumiram beber em binge e mais da metade tinham dois ou mais parceiros sexuais. A maioria dos adolescentes não procuraram os serviços de saúde $(57,21 \%)$.

A prevalência dos adolescentes que procuram por um serviço de saúde foi maior: a) no sexo feminino; b) em adolescentes cujas mães tivessem escolaridade mínima de 9 anos; c) em adolescen- 
tes fisicamente ativos; d) em adolescentes cujos responsáveis não recebiam bolsa família (Tabela 1).

Através da análise multinível, observou-se que as variáveis contextuais (modalidade de ensino da escola e o IVS) não influenciaram a procura por serviços de saúde entre os adolescentes no segundo modelo (Tabela 2). No terceiro modelo, que analisou as variáveis individuais, observa-se que apenas o sexo feminino $(\mathrm{OR}=1,80)$, ter mãe com escolaridade maior ou igual a 9 anos de estudo $(\mathrm{OR}=1,30)$, não receber bolsa família $(\mathrm{OR}=1,23)$ e ser ativo fisicamente $(\mathrm{OR}=1,32)$ foram associados a uma maior procura por serviços ou profissionais de saúde. No quarto e último modelo, as variáveis contextuais continuaram sem apresentar associação significativa com a procura pelos serviços, e as mesmas variáveis individuais que apresentaram associação no terceiro modelo também foram associadas neste último.
Apesar das variáveis uso de álcool em binge e autopercepção de saúde não terem apresentado associação significativa na análise bivariada, optamos por inclui-las no modelo multinível devido à importância dessas variáveis considerando as crenças em saúde e as necessidades em saúde da teoria comportamental de Andersen.

À medida em que acrescentamos mais variáveis no modelo, diminuiu-se a heterogeneidade entre as escolas, atingindo um MOR de $\mathbf{1}$.

\section{Discussão}

Estudos realizados em diferentes países têm demonstrado percentuais de procura e utilização de serviços de saúde distintos uns dos outros ${ }^{13-16}$. Além da especificidade dos serviços, as diferenças entre os países também são evidentes, pois enquanto em uma pesquisa realizada com uma amostra nacional no Brasil foi demonstrado que

Tabela 1. Associação bivariada das variáveis independentes e procura por serviços de saúde pelos adolescentes de escolas públicas. Olinda, Pernambuco, Brasil.

\begin{tabular}{|c|c|c|c|c|c|c|c|}
\hline \multirow{3}{*}{ Variáveis } & \multirow{3}{*}{ Categorias } & \multicolumn{4}{|c|}{ Procura por serviços de saúde } & \multirow{3}{*}{ Total } & \multirow{3}{*}{$\mathbf{p}$} \\
\hline & & \multicolumn{2}{|c|}{ Não } & \multicolumn{2}{|c|}{ Sim } & & \\
\hline & & $\mathbf{n}$ & $\%$ & $\mathbf{n}$ & $\%$ & & \\
\hline \multirow[t]{2}{*}{ Sexo } & Feminino & 696 & 52,02 & 642 & 47,98 & 1.338 & 0,000 \\
\hline & Masculino & 675 & 63,92 & 381 & 36,08 & 1.056 & \\
\hline \multirow{2}{*}{ Idade } & 14 a 16 & 732 & 56,74 & 558 & 43,26 & 1.290 & 0,539 \\
\hline & 17 a 19 & 665 & 57,98 & 482 & 42,02 & 1.147 & \\
\hline Escolaridade & $<9$ anos de estudo & 434 & 60,11 & 288 & 39,89 & 722 & 0,001 \\
\hline Materna & $\geq 9$ anos de estudo & 662 & 52,58 & 597 & 47,42 & 1.259 & \\
\hline \multirow[t]{2}{*}{ Raça } & Branca & 214 & 53,50 & 186 & 46,50 & 400 & 0,127 \\
\hline & Não Branca & 1.163 & 57,63 & 855 & 42,37 & 2.018 & \\
\hline \multirow{2}{*}{ Binge driking } & Não & 961 & 58,17 & 691 & 41,83 & 1.652 & 0,125 \\
\hline & $\operatorname{Sim}$ & 423 & 54,86 & 348 & 45,14 & 771 & \\
\hline \multirow[t]{2}{*}{ Cigarro } & Não & 1.231 & 57,31 & 917 & 42,69 & 2.148 & 0,839 \\
\hline & $\operatorname{Sim}$ & 149 & 56,65 & 114 & 43,35 & 263 & \\
\hline \multirow[t]{2}{*}{ Atividade física } & Ativo & 245 & 52,69 & 220 & 47,31 & 465 & 0,030 \\
\hline & Inativo & 1.147 & 58,22 & 823 & 41,78 & 1.970 & \\
\hline \multirow{2}{*}{$\begin{array}{l}\text { No de parceiros } \\
\text { sexuais }\end{array}$} & 1 ou nenhum & 249 & 57,77 & 182 & 42,23 & 431 & 0,569 \\
\hline & 2 ou mais & 447 & 56,09 & 350 & 43,91 & 797 & \\
\hline \multirow[t]{2}{*}{ Renda mensal } & Mais que um salário mínimo & 324 & 48,87 & 339 & 51,13 & 663 & 0,000 \\
\hline & Até um salário mínimo & 539 & 58,14 & 388 & 41,86 & 927 & \\
\hline \multirow[t]{2}{*}{ Bolsa família } & Sim & 658 & 60,70 & 426 & 39,30 & 1.084 & 0,001 \\
\hline & Não & 695 & 53,75 & 598 & 46,25 & 1.293 & \\
\hline \multirow[t]{2}{*}{$\begin{array}{l}\text { Autopercepção } \\
\text { de saúde }\end{array}$} & $\begin{array}{l}\text { Nada saudável / Não muito } \\
\text { saudável }\end{array}$ & 441 & 57,65 & 324 & 42,35 & 765 & 0,797 \\
\hline & $\begin{array}{l}\text { Saudável / Bastante saudável / } \\
\text { Completamente saudável }\end{array}$ & 962 & 57,09 & 723 & 42,91 & 1685 & \\
\hline
\end{tabular}

Fonte: elaborada pelas autoras. 
Tabela 2. Resultados do modelo de análise multinível para a procura por serviços de saúde pelos adolescentes de escolas públicas. Olinda, Pernambuco, Brasil.

\begin{tabular}{|c|c|c|c|c|}
\hline & $\begin{array}{c}\text { Modelo } \\
\text { Vazio } \\
\text { (Modelo 1) }\end{array}$ & $\begin{array}{l}\text { Interceptação } \\
\text { aleatória, } \\
\text { variáveis } \\
\text { contextuais de } \\
\text { efeitos fixos } \\
\text { (Modelo 2) }\end{array}$ & $\begin{array}{l}\text { Interceptação } \\
\text { aleatória, } \\
\text { variáveis } \\
\text { individuais de } \\
\text { efeitos fixos } \\
\text { (Modelo 3) }\end{array}$ & $\begin{array}{c}\text { Interceptação } \\
\text { aleatória, } \\
\text { variáveis } \\
\text { (individuais } \\
\text { contextuais) } \\
\text { de efeito fixo } \\
\text { (Modelo 4) }\end{array}$ \\
\hline Parte fixa & $\begin{array}{r}0,74^{* * *} \\
{[0,66-0,84]}\end{array}$ & $\begin{array}{r}0,64^{*} \\
{[0,41-1,00]}\end{array}$ & $\begin{array}{r}0,36^{* * *} \\
{[0,27-0,48]}\end{array}$ & $\begin{array}{r}1,07 \\
{[0,66-1,76]}\end{array}$ \\
\hline \multicolumn{5}{|l|}{ Fatores individuais } \\
\hline Feminino versus Masculino & & & $\begin{array}{r}1,80^{* * *} \\
{[1,48-2,19]}\end{array}$ & $\begin{array}{r}0,56^{* * *} \\
{[0,46-0,68]}\end{array}$ \\
\hline $\begin{array}{l}\text { Escolaridade materna } \\
\geq 9 \text { anos de estudo versus }<9 \text { anos }\end{array}$ & & & $\begin{array}{r}1,30^{*} \\
{[1,06-1,59]}\end{array}$ & $\begin{array}{r}0,79^{*} \\
{[0,64-0,96]}\end{array}$ \\
\hline Bolsa Família (não versus sim) & & & $\begin{array}{r}1,23^{*} \\
{[1,02-1,49]}\end{array}$ & $\begin{array}{r}1,21^{*} \\
{[1,00-1,47]}\end{array}$ \\
\hline Binge (sim versus não) & & & $\begin{array}{r}1,08 \\
{[0,88-1,32]}\end{array}$ & $\begin{array}{r}1,08 \\
{[0,89-1,32]}\end{array}$ \\
\hline $\begin{array}{l}\text { Atividade física } \\
\text { (ativo versus inativo) }\end{array}$ & & & $\begin{array}{r}1,32^{*} \\
{[1,04-1,68]}\end{array}$ & $\begin{array}{r}0,76^{*} \\
{[0,60-0,96]}\end{array}$ \\
\hline $\begin{array}{l}\text { Autopercepção (saudável/ bastante saudável/ } \\
\text { completamente saudável versus nada saudável/ } \\
\text { não muito saudável) }\end{array}$ & & & $\begin{array}{r}1,19 \\
{[0,97-1,46]}\end{array}$ & $\begin{array}{r}0,84 \\
{[0,68-1,03]}\end{array}$ \\
\hline \multicolumn{5}{|l|}{$\begin{array}{l}\text { Fatores contextuais } \\
\text { (Nível escolar) }\end{array}$} \\
\hline Período semi-integral / Período integral versus & & 0,81 & & 0,88 \\
\hline Período regular & & {$[0,66-1,00]$} & & {$[0,72-1,06]$} \\
\hline $\begin{array}{l}\text { Índice de vulnerabilidade social do bairro } \\
\text { (alto/muito alto versus médio/baixo/muito } \\
\text { baixo) }\end{array}$ & & $\begin{array}{r}2,31 \\
{[0,66-8,05]}\end{array}$ & & $\begin{array}{r}2,29 \\
{[0,71-7,41]}\end{array}$ \\
\hline \multicolumn{5}{|l|}{ Parte aleatória } \\
\hline $\begin{array}{l}\text { Variação no nível da área (Interceptação } \\
\text { aleatória) }\end{array}$ & $\begin{array}{r}0,22 \\
{[0,13-0,38]}\end{array}$ & $\begin{array}{r}0,16 \\
{[0,07-0,37]}\end{array}$ & $\begin{array}{r}0,07 \\
{[0,002-3,17]}\end{array}$ & $2,91 \mathrm{e}-08$ \\
\hline $\mathrm{PCV}^{\&}$ & & $-27,3 \%$ & $-68,2 \%$ & $-99,9 \%$ \\
\hline Median Odds Ratio & $\begin{array}{l}\text { MOR = } \\
1,23420\end{array}$ & $\begin{array}{l}\text { MOR = } \\
1,16792\end{array}$ & $\begin{array}{l}\mathrm{MOR}= \\
1,07166\end{array}$ & $\begin{array}{c}\mathrm{MOR}= \\
1\end{array}$ \\
\hline Observações & 2.454 & 2.454 & 1.842 & 1.842 \\
\hline
\end{tabular}

Fonte: elaborada pelas autoras.

apenas $48 \%$ procuraram algum serviço ou profissional de saúde para atendimento para sua própria saúde, nos Estados Unidos, esse percentual chegou a $94 \%{ }^{13,14}$.

É importante, ainda, considerar as diferenças existentes entre os sistemas de saúde em diferentes países. Nos Estados Unidos, por exemplo, os programas públicos de proteção à saúde dirigemse apenas aos mais necessitados. Assim, aqueles que não tem renda suficiente, mas não são considerados pobres, não fazem parte dos programas públicos e nem dos convênios privados de saú$\mathrm{de}^{17}$.

Neste estudo, foi observado que $42,79 \%$ dos adolescentes procuraram algum serviço ou profissional de saúde nos 12 meses anteriores à pesquisa, demonstrando uma semelhança com os dados brasileiros e que são necessárias ações para 
aproximar o adolescente dos serviços e profissionais, para, assim, prevenir agravos à saúde.

Observou-se que a prevalência da procura por serviços foi maior entre as adolescentes do sexo feminino, algo que também foi demonstrado em outros estudos ${ }^{13,18-20}$. De modo geral, as mulheres, não só adolescentes, procuram mais por serviços de saúde. Isso pode ocorrer porque há a crença de que o homem é mais forte e não adoece, mas também porque os próprios serviços não incluem a pessoa do sexo masculino na sua rotina de trabalho. Nas agendas dos serviços da atenção primária, por exemplo, há o dia do prénatal, da realização da citologia oncótica para prevenção do câncer de colo uterino, de forma o homem não se enxerga dentro desses serviços ${ }^{21}$.

Em relação às condições socioeconômicas dos adolescentes, este estudo evidenciou que quem não recebia Bolsa Família tinha maiores chances de ter procurado por algum serviço ou profissional de saúde. Outros estudos também evidenciam a influência dessas condições sobre a procura e utilização dos serviços de saúde. No Brasil, foi observado que estudantes de escola privada tinham maiores chances de procurar os serviços do que os de escola pública ${ }^{13,19}$. Enquanto em outro estudo, também realizado no Brasil, no Rio de Janeiro, demonstrou-se que havia uma maior dificuldade em agendar consultas ginecológicas para adolescentes estudantes de escola pública estadual, quando comparadas às estudantes de escolas privadas ou federais ${ }^{22}$.

A provável explicação para isso é o menor acesso aos cuidados de saúde que tem alguém que depende exclusivamente do sistema público. Nesse estudo, apesar de não haver uma comparação entre estudantes de escolas públicas e privadas, o recebimento de Bolsa Família foi considerado um proxy de renda, o que confirma a afirmativa anterior, de que as condições econômicas interferem diretamente no acesso aos serviços de saúde.

Outros estudos realizados nos Estados Unidos e Oceania evidenciaram que os adolescentes cujos pais não podiam pagar pelas consultas tiveram menores chances de visitar um profissional de saúde ${ }^{23,24}$. Como já foi citado anteriormente, o Brasil e os Estados Unidos possuem sistemas de saúde bem diferentes. No Brasil, o princípio da universalidade do Sistema Único de Saúde (SUS) deve garantir acesso aos serviços de saúde a toda população. Entretanto, pode-se observar que em Olinda, em 2018, apenas 49,44\% da população residia em área coberta pela atenção primária, de forma que ainda se tem muito a avançar para garantir acesso universal à saúde.
Neste estudo, e em outros já realizados, foi observado que adolescentes que tinham mães com maior escolaridade procuraram mais o serviço ou profissional de saúde em comparação aos com mães com menor nível de escolaridade. Esse dado sugere que as mães, com maior nível de instrução, conseguem influenciar positivamente a atitude do filho frente a sua saúde $\mathrm{e}^{13,19,23}$.

Observou-se que os adolescentes ativos fisicamente tiveram maiores chances de procurar um serviço ou profissional de saúde em comparação aos inativos. Isso pode ser explicado, pois sendo a atividade física um fator importante na promoção da saúde e prevenção de doenças, aqueles indivíduos que a praticam, podem estar mais atentos e preocupados com a sua saúde, buscando orientação e cuidados periódicos com profissionais. Um estudo realizado com adolescentes brasileiros evidenciou que a procura por serviços de saúde foi menor entre aqueles que não faziam nada em relação ao próprio peso corporal, o que corrobora com esses dados ${ }^{19}$.

Não foram encontradas diferenças estatisticamente significantes na associação entre a faixa etária, a raça/cor de pele, o uso de cigarro, o número de parceiros sexuais e a autopercepção de saúde com a procura por serviços de saúde entre adolescentes neste estudo.

Realizamos uma análise em relação ao regime escolar, comparando os estudantes de escolas com regime integral, semi-integral e regular, e não foram encontradas diferenças estatisticamente significantes.

Além disso, o contexto escolar, analisado através do IVS, não foi importante para explicar a procura por serviços de saúde. Em relação ao IVS, nesta pesquisa ele refere-se ao local onde a escola está situada que não necessariamente é o local onde o estudante reside, isso pode explicar a não associação com a procura pelos serviços.

Por outro lado, um estudo realizado na região metropolitana de São Paulo evidenciou que o local de residência não apresentou associação com o uso dos serviços de saúde pela população, logo não configura uma barreira significante ${ }^{25}$.

Como limitação do estudo, é possível citar algo próprio do tipo de estudo transversal, no qual as variáveis de exposição e desfecho são pesquisadas em um mesmo período, correspondente à coleta de dados. Dessa forma, não se pode inferir a relação causal entre as variáveis. Além disso, a amostra do estudo não foi selecionada considerando a análise contextual e, por isso, um mesmo bairro teve mais de uma escola, isto é, três bairros tiveram duas ou três escolas, quando 
deveria ter apenas uma. Tivemos 27 escolas e 22 bairros. Assim, são necessários mais estudos que analisem a associação da procura por serviços ou profissionais de saúde com o contexto social no qual o adolescente está inserido.

Também se pode citar que pode ter ocorrido o viés de memória, uma vez que algumas respostas estavam condicionadas à lembrança dos ado- lescentes sobre fatos que ocorreram no período de um ano. Além desse viés, o de resposta também pode ter acontecido, devido ao constrangimento e ao medo que alguns indivíduos podem ter sentido em relação à confidencialidade das informações, mesmo que essa confidencialidade tenha sido garantida pelos pesquisadores.

\section{Colaboradores}

AMCL Peixoto, TQ Melo e LAA Ferraz contribuíram com a aquisição, análise e interpretação dos dados e a redação do manuscrito. CFBF Santos, F Godoy, PAM Valença, VA Menezes e V Colares contribuíram com a elaboração e delineamento do estudo, análise a interpretação dos dados e revisão do manuscrito.

\section{Financiamento}

Coordenação de Aperfeiçoamento de Pessoal de Nível Superior (CAPES) 


\section{Referências}

1. Assis SG, Avanci JQ, Duarte CS. Adolescência e saúde coletiva: entre o risco e o protagonismo juvenil. Cien Saude Colet 2015;20(11):3296-3296.

2. Stopa SR, Malta DC, Monteiro CN, Szwarcwald CL, Goldbaum M, Cesar CLC. Acesso e uso de serviços de saúde pela população brasileira, Pesquisa Nacional de Saúde 2013. Rev Saude Publica 2017;51(1):1S-11S.

3. Travassos C, Martins M. Uma revisão sobre os conceitos de acesso e utilização de serviços de saúde. Cad Saude Publica 2004; 20(2):190-198.

4. Puente-Palacios KE, Laros JA. Análise multinível: contribuições para estudos sobre efeito do contexto social no comportamento individual. Estud Psicol 2009; 26(3):349-361.

5. Andersen RM. Revisiting the Behavioral Model and Access to Medical Care: Does it Matter? J Health Soc Behav 1995; 36(10):1-10.

6. Pavão ALB, Coeli CM. Theoretical models of health services utilization:concepts and review. Cad Saude Colet 2008; 16(3):471-481.

7. Guedes DP, Lopes CC. Validação da versão brasileira do Youth Risk Behavior Survey 2007. Rev Saude Publica 2010; 44(5):840-850.

8. Oliveira MM de, Campos MO, Andreazzi MAR de, Malta DC. Características da Pesquisa Nacional de Saúde do Escolar - PeNSE. Epidemiol Serv Saude 2017; 26(3):605-616.

9. Gomes DJL. Educação integral no ensino médio: uma análise da proposta curricular das Escolas de Referência em Ensino Médio na perspectiva transdisciplinar. Revista Cadernos de Estudos e Pesquisa na Educação Básica 2017; 3(1):137-158

10. Instituto de Pesquisa Econômica Aplicada (IPEA). Vulnerabilidade social no Brasil: conceitos, métodos e primeiros resultados para Municípios e regiões metropolitanas brasileiras. Rio de Janeiro: IPEA; 2018. (Texto para Discussão, n. 2364)

11. Austin PC, Merlo J. Intermediate and advanced topics in multilevel logistic regression analysis. Stat Med 2017;36(20):3257-3277.

12. Merlo J, Yang M, Chaix B, Lynch J, Råstam L. A brief conceptual tutorial on multilevel analysis in social epidemiology: Investigating contextual phenomena in different groups of people. J Epidemiol Community Health 2005; 59(9):729-736.

13. Oliveira MM, Andrade SSCA, Campos MO, Malta DC. Fatores associados à procura de serviços de saúde entre escolares brasileiros: uma análise da Pesquisa Nacional de Saúde do Escolar (PeNSE), 2012. Cad Saude Publica 2015; 31(8):1603-1614.

14. Secor-Turner MA, Randall BA, Brennan AL, Anderson MK, Gross DA. Rural Adolescents' Access to Adolescent Friendly Health Services. J Pediatr Heal Care 2014; 28(6):534-540.

15. Bilal SM, Spigt M, Dinant GJ, Blanco R. Sexual \& Reproductive Healthcare Utilization of Sexual and Reproductive Health Services in Ethiopia - Does it affect sexual activity among high school students? Sex Reprod Health 2015; 6(1):14-18.
16. Dagnew T, Tessema F, Hiko D. Health service utilization and reported satisfaction among adolescents in dejen district, ethiopia: a cross-sectional study. Ethiop J Health Sci 2009; 25(1):17-28.

17. Marques RM, Piola SF, Carrillo Roa A. Sistema de Saúde no Brasil: organização e financiamento. 2016. 264 p.

18. Kekkonen VK, Kivimaki P, Valtonen H, Tolmunen T, Lehto SM, Hintikka J, Laukkanen E. Psychosocial problems in adolescents associated with frequent health care use. Fam Pract 2015; 32(3):305-310.

19. Oliveira MM, Andrade SSCA, Stopa SR, Malta DC. Demand for health services or professionals among Brazilian adolescents according to the National School Health Survey 2015. Rev Bras Epidemiol 2018; 21(Supl. 1):E180003.

20. Nunes BP, Flores TR, Duro SMS, Saes MO, Tomasi E, Santiago AD, Thumé E, Facchini LA. Utilização dos serviços de saúde por adolescentes: estudo transversal de base populacional, Pelotas-RS, 2012. Epidemiol Serv Saude 2015; 24(250):411-420.

21. Levorato CD, Mello LM, Silva AS, Nunes AA. Fatores associados à procura por serviços de saúde numa perspectiva relacional de gênero. Cien Saude Colet 2014; 19(4):1263-1274.

22. Pereira SM, Taquette SR, Pérez MA. Study of the sexuality and demand for gynecologic services by high school students in the city of Rio de Janeiro. J Pediatr Adolesc Gynecol 2013; 26(6):323-326.

23. Aalsma MC, Gilbert AL, Xiao S, Rickert VI. Parent and Adolescent Views on Barriers to Adolescent Preventive Health Care Utilization. J Pediatr 2016;169:140145.

24. Kennedy EC, Bulu S, Harris J, Humphreys D, Malverus J, Gray NJ. "Be kind to young people so they feel at home": a qualitative study of adolescents' and service providers? perceptions of youth-friendly sexual and reproductive health services in Vanuatu. BMC Health Serv Res 2013 Dec 31; 13(1):455.

25. Chiavegatto Filho AD, Wang YP, Malik AM, Takaoka J, Viana MC, Andrade LH. Determinants of the use of health care services: multilevel analysis in the Metropolitan Region of Sao Paulo. Rev Saude Publica 2015; 49:15.

Artigo apresentado em 24/05/2020

Aprovado em 14/04/2021

Versão final apresentada em 16/04/2021

Editores-chefes: Romeu Gomes, Antônio Augusto Moura da Silva 
